\title{
Influence of acute hyperlipidemia to adipocyte-derived hormones in lean normotensive and subjects with metabolic syndrome
}

\author{
Heno F Lopes ${ }^{3,4^{*}}$, Richard L Klein ${ }^{2}$, W Timothy Garvey ${ }^{2}$, Theodore Goodfriend ${ }^{5}$ and Brent M Egan ${ }^{1}$
}

\begin{abstract}
Background: Adipocyte-derived factors and regulators likely contribute to the metabolic syndrome (MetS) in patients with central obesity. This study was undertaken to assess the contribution of leptin, adiponectin, and acylation stimulating protein (ASP-C3ades/ARG) to hemodynamic (blood pressure [BP]) and metabolic (insulin, glucose, lipids) features of MetS.

Methods: In this study, leptin, adiponectin, and C3ades/ARG were measured at baseline and in response to an infusion of Intralipid ${ }^{\Phi}$ and heparin in 12 lean healthy controls and 12 patients with MetS.

Results: Baseline plasma leptin ( $27.6 \pm 6.2$ vs. $10.9 \pm 3.8 \mathrm{ng} / \mathrm{mL}, \mathrm{p}<0.01)$ and plasma C3ades/ARG $(273 \pm 79 \mathrm{vs}$ $198 \pm 57 \mathrm{mg} / \mathrm{dL}, \mathrm{p}<0.05)$ were higher in the MetS than control group, whereas baseline plasma adiponectin was higher in the control than MetS group $(9.9 \pm 1.9$ vs. $5.4 \pm 0.6 \mathrm{~g} / \mathrm{mL}$ ). Plasma leptin correlated with body mass index $(\mathrm{BMI})$, systolic and diastolic BP $(r=0.53-0.77, \mathrm{p}<0.01)$. Conversely, adiponectin correlated inversely with insulin, glucose, waist circumference, and insulin sensitivity $(r=0.48-0.51, p \leq 0.02)$. Plasma triglycerides increased similarly in MetS and control groups after 4-hours of Intralipid and heparin. C3ades/ARG increased only in lean volunteers. The decrease in triglycerides 1-hour post-infusion was lower in the MetS than control group $(-116 \pm 33$ vs. $-282 \pm 81 \mathrm{mg} / \mathrm{dL}, \mathrm{p}=0.01)$ and correlated inversely with the change in C3ades/ARG.
\end{abstract}

Conclusion: These data suggest that leptin is more closely associated with hemodynamic (BP) aspects of MetS, whereas adiponectin and C3ades/ARG are more closely associated with metabolic components.

Keywords: Intralipid ${ }^{\oplus}$ and heparin, Adiponectin, Leptin, ASP/C3adesARG, Lean normotensives, Metabolic syndrome subjects

\section{Background}

A rapidly growing body of literature indicates that adipocyte-derived signaling hormones and regulators of adipocyte function play an important role in the pathogenesis and complications of metabolic syndrome (MetS). Leptin, an adipocyte secreted hormone, has multiple cardiovascular functions and is associated with sympathetic activation, angiogenesis, oxidative stress and thrombosis [1-4]. Adiponectin, a novel adipocyte-derived collagen-like protein, has been associated linked to anti-inflammatory

\footnotetext{
* Correspondence: hipheno@gmail.com

${ }^{3}$ Unidade de Hipertensao-Heart Institute (InCor), University of Sao Paulo Medical School, Av Dr Enéas C. de Aguiar, 44, Zip code 05403-904 São Paulo, Brazil

${ }^{4}$ Universidade Nove de Julho - UNINOVE, University of São Paulo Medical School, Av Dr Enéas C. de Aguiar, 44, Zip code 05403-904 São Paulo, Brazil Full list of author information is available at the end of the article
}

and anti-atherogenic proprieties $[5,6]$. Leptin and adiponectin were inversely related in normal controls and obese women [7]. C3adesARG (ASP) is a complement C3a component resulted from the removal of the carboxy-terminal arginine. ASP/C3adesARG increases postprandially and correlated with the rate of triglyceride clearance in one study, but levels did not change post-prandially in another report $[8,9]$. ASP/C3adesARG is an adipocyte product that correlated with postprandial lipemia in subjects with coronary artery disease [10]. These observations suggest that different adipocyte-derived factors and the balance between these factors contribute to various features of MetS and its cardiovascular diseases risk factors. At least some of these factors are responsive to an acute oral and/or parenteral lipid load. The aim of this study was to evaluate the relationship of plasma leptin, adiponectin, 
and ASP/C3adesARG to various aspects of MetS and the response of these factors to acute hyperlipidemia. Subjects for this study included obese subjects with features of the MetS and age, gender, and race-matched lean, healthy volunteers.

\section{Methods \\ Subjects}

Twelve subjects with features of MetS (waist circumference $\geq 88 \mathrm{~cm}$ for women and $\geq 102 \mathrm{~cm}$ for men, triglycerides $>150 \mathrm{mg} / \mathrm{dL}$ and/or HDL-cholesterol $<45$ for women or $<40 \mathrm{mg} / \mathrm{dL}$ for men, BP $<130-159 / 85-99 \mathrm{mmHg}$ ) and 12 healthy volunteers (waist circumference $<88 \mathrm{~cm}$ for women and $<102 \mathrm{~cm}$ for men, BP $<130 / 85 \mathrm{mmHg}$ ) with normal lipids matched for age, race, and gender were evaluated as described previously [11]. In brief, subjects read and signed a written informed consent document approved by the Institutional Review Board of Medical University of South Carolina. They were scheduled for 3 consecutive weekly screening visits to ensure eligibility. Subjects were considered in MetS group when they met at least 3 criteria defined by the Adult Treatment Panel III classification [12].

\section{Physiological data}

During the qualifying visits, subjects had their blood pressure and heart rate measured in triplicate in the sitting position. Blood pressures were measured by a trained observer using a mercury sphygmomanometer and appropriately sized cuff. The first Korotkoff sound defined systolic blood pressure and the disappearance of 5 th Korotkoff sound determined diastolic blood pressure. Heart rate was measured by palpation of the radial pulse for 30 seconds between the 2 nd and $3^{\text {rd }}$ measurements of blood pressure with subjects in the seated position.

\section{Biochemical measurements}

Blood for non-esterified fatty acids (NEFAs) was drawn into prechilled Eppendorf tubes containing paraoxon [13]. Plasma was stored at $-70^{\circ} \mathrm{C}$ before analyzing the total plasma NEFAs using the 63Ni method [14].

\section{Lipids and lipoproteins}

Triglycerides were measured by fluorometric method. Total cholesterol was measured by the colorimetric method, and HDL- cholesterol was prepared from whole plasma by precipitation with phosphotungstate- $\mathrm{MgCl} 2$ [15]. LDL-cholesterol and VLDL-cholesterol were calculated [16].

\section{Adipocyte factors}

Plasma leptin was measured by radioimmunoassay method and plasma adiponectin was measured by the Elisa method. Plasma ASP/C3adesARG was measured by the Elisa sandwich Immunoassay method. Total antioxidant capacity was measured by the ferric reducing activity of plasma (FRAP) assay under fasting conditions.

\section{Total antioxidant capacity}

FRAP assay was performed as previously described [17]. FRAP activity was measured in triplicate on each sample and the mean of the three values was used in the analysis.

\section{Insulin and HOMAir}

Plasma insulin was measured by radioimmunoassay method and the homeostatic model assessment for insulin sensitivity (HOMAir) was calculated dividing glucose $(\mathrm{mmol} / \mathrm{L}) \times$ insulin (IU/L) by 22.5 .

\section{Protocol}

On the study day subjects were admitted to the outpatient General Clinical Research Center (GCRC) at 08:00 following an overnight fast. Intravenous access was established in both arms, with one side for obtaining blood samples and the other for infusion of Intralipid ${ }^{\oplus}$ and heparin. After 30 minutes of resting blood samples were drawn for serum lipids and plasma glucose, insulin, total antioxidant capacity (FRAP assay), NEFAs, triglycerides, adiponectin, and ASP/C3adesARG. An infusion was then started of $20 \%$ Intralipid $^{\circledR}$ (Baxter Healthcare Corp., Glendale, CA) at $0.8 \mathrm{ml} / \mathrm{m} 2 / \mathrm{min}$ and heparin (200 U bolus, followed by infusion at $1000 \mathrm{U} / \mathrm{h}$ ). Heparin was given to activate lipoprotein lipase and accelerate the hydrolysis of fatty acids from triglycerides. Blood samples were obtained for triglycerides, NEFAs, leptin, adiponectin, insulin, and ASP/C3adesARG at 2-hours and 4-hours of the Intralipid ${ }^{\circ}$ and heparin infusion.

\section{Statistical analysis}

Group differences in dichotomous variables including gender and race were examined using the Chi-square test. Age, casual blood pressure and heart rate were shown as median, minimum and maximum and body mass index (BMI) in media and standard error. The Student's unpaired t-test was used to compare casual blood pressure, heart rate and BMI. Changes in variables over time during the infusion of Intralipid ${ }^{\circledR}$ and heparin were assessed using the General Linear Model for repeated measures. Correlations were determined by Pearson's method. Statistical analyses were performed with the SPSS/PC 20.0 statistical software package (SPSS, Chicago, IL). A p-value $<0.05$ was accepted as statistically significant. All multiple comparisons procedures were taken into consideration after False Discovery Rate (FDR) as described by Benjamini and Hochberg [18].

\section{Results and discussion}

Demographic data for control and metabolic syndrome groups are shown in Table 1 . Values for leptin, adiponectin, 
Table 1 Demographic and biochemistry data from subjects with MS and controls

\begin{tabular}{|c|c|c|c|}
\hline Variables & $\begin{array}{l}\text { MS Group } \\
(n=12)\end{array}$ & $\begin{array}{l}\text { Controls } \\
(n=12)\end{array}$ & P-value \\
\hline \multirow[t]{2}{*}{ Age (years) } & 35 & 38 & NS \\
\hline & $28-46$ & $26-47$ & \\
\hline Gender (f/m) & $6 / 6$ & $6 / 6$ & NS \\
\hline Race & $7 \mathrm{AA}, 5 \mathrm{C}$ & $6 \mathrm{AA}, 6 \mathrm{C}$ & NS \\
\hline \multirow[t]{2}{*}{ Systolic BP (mm Hg) } & 132 & 111 & $<0.0001$ \\
\hline & $(130-144)$ & $(100-126)$ & \\
\hline \multirow[t]{2}{*}{ Diastolic BP (mm Hg) } & 91 & 74 & $<0.0001$ \\
\hline & $(86-94)$ & $(64-82)$ & \\
\hline \multirow[t]{2}{*}{ Heart Rate (bpm) } & 77 & 71 & $<0.005$ \\
\hline & $(72-84)$ & $(60-80)$ & \\
\hline BMI $\left(\mathrm{kg} / \mathrm{m}^{2}\right)$ & $34.1 \pm 1.9$ & $22.7 \pm 0.5$ & $<0.0001$ \\
\hline Waist Circumference (cm) & $106 \pm 4$ & $78 \pm 3$ & $<0.0001$ \\
\hline Total-cholesterol (mg/dL) & $229 \pm 6$ & $173 \pm 7$ & $<0.0001$ \\
\hline HDL-cholesterol (mg/dL) & $47 \pm 3$ & $61 \pm 5$ & $<0.05$ \\
\hline LDL-cholesterol (mg/dL) & $152 \pm 5$ & $95 \pm 6$ & $<0.0001$ \\
\hline VLDL-cholesterol (mg/dL) & $29 \pm 4$ & $16 \pm 1$ & $<0.01$ \\
\hline Triglycerides (mg/dL) & $171 \pm 30$ & $81 \pm 6$ & 0.01 \\
\hline Glucose (mg/dL) & $90 \pm 3$ & $83 \pm 3$ & 0.08 \\
\hline Insulin (UI/L) & $12.5 \pm 1$ & $6.5 \pm 0.9$ & 0.01 \\
\hline Sodium (mmol/L) & $139.3 \pm 0.7$ & $142.0 \pm 0.7$ & 0.01 \\
\hline Potassium (mmol/L) & $4.34 \pm 0.15$ & $4.32 \pm 0.08$ & NS \\
\hline
\end{tabular}

Data presented as median, minimum, maximum and mean \pm SEM.

and ASP/C3adesARG at baseline and during the Intralipid ${ }^{\circ}$ and heparin infusion in MetS and control groups are shown in (Figure 1). As shown, baseline leptin and ASP/C3adesARG was higher and adiponectin lower in the MetS than the control group (Figure 1). Plasma leptin and adiponectin did not change during Intralipid and heparin infusion in both groups. However, plasma ASP/C3adesARG increased significantly in the control group after 2-hours of Intralipid ${ }^{\oplus}$ and heparin infusion. The control group also showed a positive correlation between the changes in ASP/C3adesARG and triglycerides after 2-hours of Intralipid ${ }^{\circ}$ and heparin infusion $(r=0.80$, $p=0.002$ ). The MetS group showed a negative correlation between the changes for triglycerides and insulin during the Intralipid ${ }^{\circ}$ and heparin infusion $(r=0.80, p=0.002$; $r=-0.65, p=0.02$ for 2 and 4-hours respectively).

The correlations in all subjects combined between plasma leptin and body mass index $(\mathrm{r}=0.77, \mathrm{p}=0.001)$, systolic BP $(r=0.73, p=0.001)$, and diastolic BP $(r=57$, $\mathrm{p}=0.030$ ) were significant. The correlation between leptin and waist circumference, and heart rate did not achieve significance after FDR correction. Adiponectin correlated inversely with waist circumference $(\mathrm{r}=0.63, \mathrm{p}=0.008)$. Adiponectin correlated negatively with insulin, glucose,
HOMAir $(\mathrm{r}=0.50-0.63, \mathrm{p}<0.02)$ and positively with the total antioxidant capacity $(\mathrm{r}=0.50, \mathrm{P}<0.02)$. We found a positive correlation between plasma ASP/C3adesARG and BMI, insulin, HOMAir, and heart rate. After FDR correction these correlations did not achieve significance.

Plasma NEFAs and triglyderides increased similarly in MetS and control groups after 4-hours of Intralipid ${ }^{\circ}$ and heparin infusion. However, the decrease in triglycerides 1-hour post-infusion was significantly lower in the MetS group compared to the control group (116 \pm 33 versus $282 \pm 81 \mathrm{mg} / \mathrm{dL}, \mathrm{p}=0.01$ ).

In this study, subjects with MetS had higher plasma leptin, ASP/C3adesARG and lower adiponectin then the normal subjects (Figure 1).

These three adipocyte-derived factors showed a differential relationship to various components of MetS as described above. The findings are consistent with previous studies, which suggest that adipocytes play an important role in the pathogenesis of MetS and its complications. Collectively, evidence indicates that the level of and balance between specific adipocyte-derived peptides and lipids may modulate the expression of the hemodynamic and metabolic components of the insulin resistance syndrome.

Leptin has been implicated as a key transducing factor between obesity and hypertension [19]. Leptin's pressor effects appear to be mediated in large part by activation of the sympathetic nervous system. While insulin and leptin are both elevated with obesity and both activate the nervous system, the actions of leptin appear to predominate $[20,21]$. In this study, we found a positive correlation between plasma leptin and body mass index, systolic BP, and diastolic BP. These findings are consonant with those of Leyva, et al, who showed a positive correlation between leptin and BMI, systolic and diastolic BP [22]. Another important results in this study are related to the negative correlation between adiponectin insulin, glucose, HOMAir and waist circumference. These results suggest a negative correlation between insulin resistance and adiponectin. Since adiponectin, is an adipocyte-derived collagenlike protein that has been associated to anti-inflammatory and anti-atherogenic proprieties, it makes sense that subjects with features of insulin resistance, a cardiovascular risk factor, are supposed to show lower plasma adiponectin levels. We also found a positive correlation between adiponectin and the total antioxidant capacity (FRAP assay), although it disappeared after FDR correction. It suggests that the highest the total antioxidant capacity better are the anti-atherogenic and anti-inflammatory profiles.

Maslowska and coworkers showed a higher plasma ASP/C3adesARG in obese than non-obese subjects and suggested that ASP/C3adesARG pathway has involvement in the pathogenesis of obesity [23]. Since subjects with the features of MetS have a high triglycerides level comparing to normal subjects and the ASP/C3adesARG is associated 

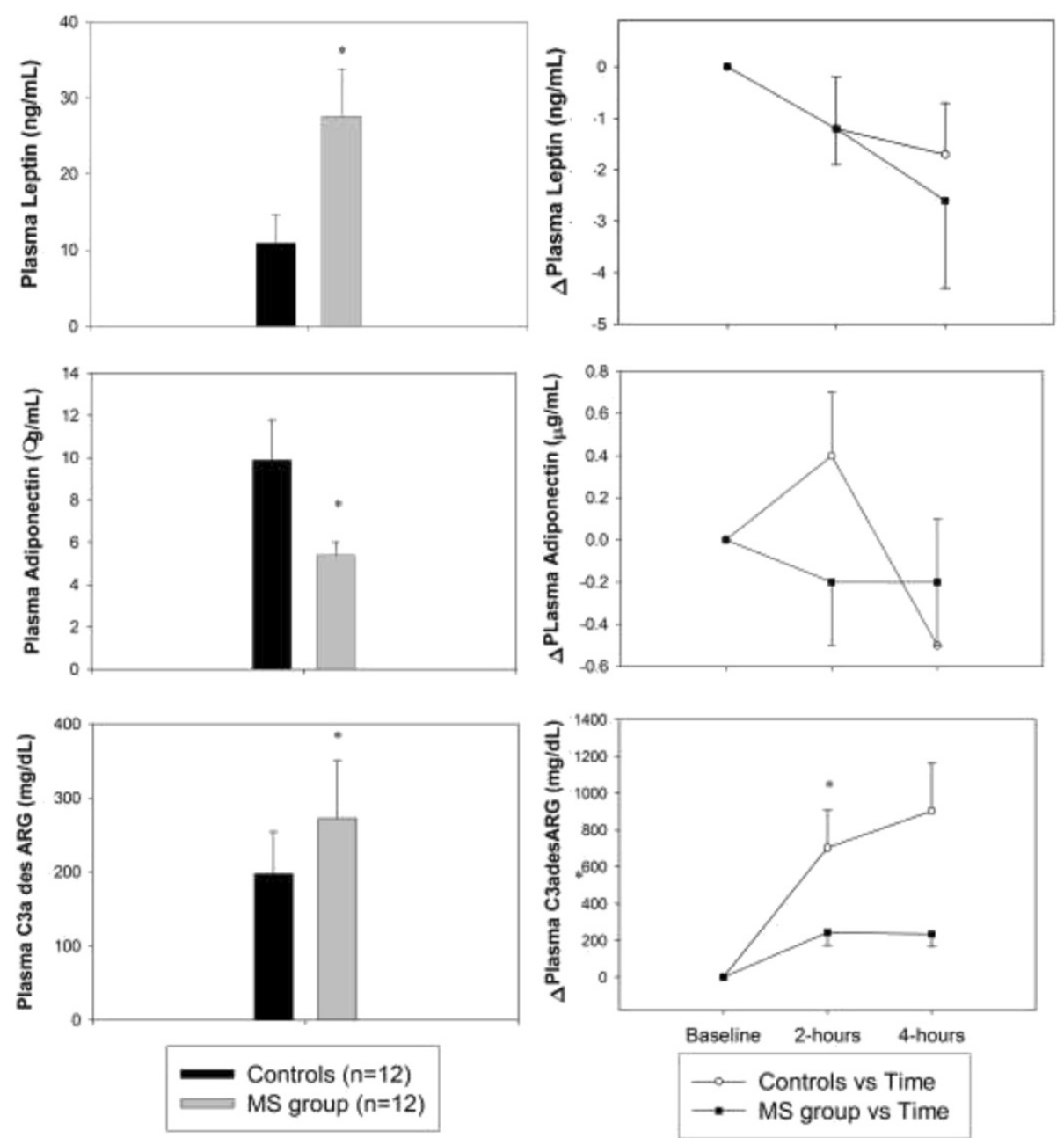

Figure 1 Plasma leptin, adiponectin and ASP/C3adesARG values at baseline and after Intralipid ${ }^{\circledR}$ infusion in control and metabolic syndrome groups. ${ }^{*} \mathrm{p}<0.05$.

to triglycerides clearance, it suggest that the higher plasma level of ASP/C3adesARG in obese subjects can be a sign of a malfunctioning mechanism of this protein. Plasma ASP/C3adesARG increased significantly in control group during Intralipid ${ }^{\circ}$ and heparin infusion. Meijssen et al showed an increased post-prandial ASP/C3adesARG response in subjects with familial combined hyperlipidemia [24]. Halkes et al. also showed an increase in plasma ASP/ C3adesARG after oral lipid load in normolipemic subjects with coronary artery disease and normal volunteers [10]. Although this two studies showed an increase in C3adesARG in humans exposed to an oral lipid load, the results over the literature is not concordant. For instance, Charlesworth et al did not find any change to postprandial plasma ASP/C3adesARG in healthy subjects submitted to an oral lipid load [9]. Even though, the authors observed an important individual variation in postprandial plasma ASP/C3adesARG levels in their study. In our study, the changes in plasma ASP/C3adesARG also correlated to changes on triglycerides in control subjects during Intralipid ${ }^{\circ}$ and heparin infusion. The postprandial triglycerides clearance was delayed in MetS comparing to control group. In experimental study, Murray et al. already showed delayed post-prandial triglycerides clearance in ASP-deficient mice comparing to wild type [25]. ASP/ C3adesARG is already known as a complement 3 fragment related to triglycerides clearance [8]. However, this is the first time that evidence of a blunted release of ASP/ C3adesARG in subjects with the cluster of cardiovascular risk factors submitted to an Intralipid ${ }^{\bullet}$ and heparin infusion, a model of acute hyperlipidemia, is showed. Since MetS group, which has higher fasting plasma triglycerides, did not show any increase in ASP/C3adesARG during Intralipid $^{\circ}$ and heparin infusion and also showed lower triglycerides clearance after an Intralipid ${ }^{\circ}$ and heparin infusion, we can speculate a possible deficiency of ASP/ C3adesARG synthesis in MetS subjects exposed to an acute hyperlipidemia. 


\section{Conclusions}

In conclusion, subjects with features of MetS have higher leptin, ASP/C3adesARG, and lower plasma level of adiponectin. They also showed lower triglycerides clearance and ASP/C3adesARG responses to an acute hyperlipidemia. These findings, taken together with previous reports, suggest that these adipocyte-derived factors play an important role in the pathogenesis and complications of MetS. The results further suggest that the levels of and balance between the various adipocyte derived factors may modulate the expression of various metabolic and hemodynamic facets of the syndrome. The differential impact of various adipocyte factors on individual components of the syndrome raises the potential for additional therapeutic targets.

\section{Abbreviations \\ MetS: Metabolic syndrome; ASP-C3ades/ARG: Acylation stimulating protein; BP: Blood pressure; BMI: Body mass index; NEFAs: Non-esterified fatty acids; FRAP: Ferric reducing activity of plasma; HOMAir: Homeostatic model assessment for insulin sensitivity; GCRC: General Clinical Research Center.}

\section{Competing interests}

The authors declare that they have no competing interests.

\section{Authors' contributions}

HFL - acquisition, analysis and interpretation of data. RLK - analysis and interpretation of data. WTG - analysis and interpretation of data. TG - analysis and interpretation of data. BME - critical revise for important intellectual content. All authors read and approved the final manuscript

\section{Acknowledgements}

The research was supported by the National Institutes of Health HL58794, HL04290, HL55782, GM42056, DK48831, DK26657, CA77839 and by General Clinical Research Center RR-01070 from the Division of Research Resources.

\section{Author details}

'Department of Medicine, Medical University of South Carolina, Charleston, South Carolina, USA. ${ }^{2}$ Department of Pharmacology, Medical University of South Carolina, Charleston, South Carolina, USA. ${ }^{3}$ Unidade de

Hipertensao-Heart Institute (InCor), University of Sao Paulo Medical School, Av Dr Enéas C. de Aguiar, 44, Zip code 05403-904 São Paulo, Brazil.

${ }^{4}$ Universidade Nove de Julho - UNINOVE, University of São Paulo Medical School, Av Dr Enéas C. de Aguiar, 44, Zip code 05403-904 São Paulo, Brazil. ${ }^{5}$ Department of Medicine and Pharmacology, William S. Middleton Veterans Hospital University of Wisconsin, Madison, Wisconsin, USA.

Received: 19 May 2014 Accepted: 20 November 2014

Published: 2 December 2014

\section{References}

1. Haynes WG: Interaction between leptin and sympathetic nervous system in hypertension. Curr Hypertens Rep 2000, 2:311-318.

2. Park HY, Kwon HM, Lim HJ, Hong BK, Lee JY, Park BE, Jang Y, Cho SY, Kim HS: Potential role of leptin in angiogenesis: leptin induces endothelial cell proliferation and expression of matrix metalloproteinases in vivo and in vitro. Exp Mol Med 2001, 33:95-102.

3. Matsubara M, Chiba H, Maruoka S, Katayose S: Elevated serum leptin concentrations in women with components of multiple risk factor clustering syndrome. J Atheroscler Thromb 2000, 7:231-237.

4. Maruyama I, Nakata M, Yamaji K: Effect of leptin in platelet and endothelial cells. Obesity and arterial thrombosis. Ann N Y Acad Sci 2000, 902:315-319.

5. Arita $Y$, Kihara S, Ouchi N, Maeda K, Kuriyama H, Okamoto $Y$, Kumada M, Hotta K, Nishida M, Takahashi M, Nakamura T, Shimomura I, Muraguchi M, Ohmoto Y, Funahashi T, Matsuzawa Y: Adipocyte-derived plasma protein adiponectin acts as a platelet-derived growth factor-BB-binding protein and regulates growth factor-induced common postreceptor signal in vascular smooth muscle cell. Circulation 2002, 105:2893-2898.

6. Ouchi N, Arita Y, Kihara S, Nishida M, Matsuyama A, Okamoto Y, Ishigami M, Kuriyama H, Kishida K, Nishizawa H, Hotta K, Muraguchi M, Ohmoto Y, Yamashita S, Funahashi T, Matsuzawa Y: Adipocyte-derived plasma protein, adiponectin, suppresses lipid accumulation and class $A$ scavenger receptor expression in human monocyte derived macrophages. Circulation 2001, 103:1057-1063.

7. Matsubara M, Maruoka S, Katayose S: Inverse relationship between plasma adiponectin and leptin concentrations in normal-weight and obese women. Eur J Endocrinol 2002, 147:173-180.

8. Cianflone K, Vu H, Walsh M, Baldo A, Sniderman A: Metabolic response of Acylation Stimulating Protein to an oral fat load. J Lipid Res 1989, 30:1727-1733

9. Charlesworth JA, Peake PW, Campbell LV, Pussell BA, O'Grady S, Tzilopoulos T: The influence of oral lipid loads on acylation stimulating protein (ASP) in healthy volunteers. Int J Obes Relat Metab Disord 1998, 22:1096-1102.

10. Halkes CJ, van Dijk H, de Jaegere PP, Plokker HW, van Der Helm Y, Erkelens DW, Castro CM: Postprandial increase of complement component 3 in normolipidemic patients with coronary artery disease: effects of expandeddose simvastatin. Arterioscler Thromb Vasc Biol 2001, 21:1526-1530.

11. Lopes HF, Martin KL, Nashar K, Morrow JD, Goodfriend TL, Egan BM: DASH diet lowers blood pressure and lipid-induced oxidative stress in obesity. Hypertension 2003, 41:422-430.

12. Pasternak RC: Report of the Adult Treatment Panel III: the 2001 National Cholesterol Education Program guidelines on the detection, evaluation and treatment of elevated cholesterol in adults. Cardiol Clin 2003, 21(3):393-398

13. Zambon A, Hashimoto SI, Brunzell JD: Analysis of techniques to obtain plasma for measurement of levels of free fatty acids. J Lipid Res 1993, 34:1021-1028.

14. Barash H, Akov S: Improved $63 \mathrm{Ni}$ radiochemical assay of free fatty acids in plasma. Clin Chem 1987, 33:176-179.

15. De Bruin TW, Brouwer CB, Gimpel JA, Erkelens DW: Postprandial decrease in $\mathrm{HDL}$ - cholesterol and $\mathrm{HDL}$ apo $\mathrm{A}-\mathrm{I}$ in normal subjects in relation to triglyceride metabolism. Am J Physiol 1991, 260(3 Pt 1):E492-E498.

16. Friedewald WT, Levy RI, Fredrickson DS: Estimation of the concentration of lowdensity lipoprotein cholesterol in plasma, without use of the preparative ultracentrifuge. Clin Chem 1972, 18:499-502.

17. Benzie IF, Strain JJ: The ferric reducing ability of plasma (FRAP) as a measure of "antioxidant power": the FRAP assay. Anal Biochem 1996, 239:70-76.

18. Benjamini $Y$, Hochberg $Y$ : Controlling the false discovery rate: a practical and powerful approach to multiple testing. J R Stat Soc Ser B Methodol $1995,57: 89.300$

19. Mark AL, Correia ML, Rahmouni K, Haynes WG: Selective leptin resistance: a new concept in leptin physiology with cardiovascular implications. J Hypertens 2002, 20:1245-1250.

20. Landsberg L: Insulin-mediated sympathetic stimulation: role in the pathogenesis of obesity-related hypertension (or, how insulin affects blood pressure, and why). J Hypertens 2001, 19(3 Pt 2):523-528.

21. Masuo K, Mikami H, Itoh M, Ogihara T, Tuck ML: Sympathetic activity and body mass index contribute to blood pressure levels. Hypertens Res 2000 23:303-310.

22. Leyva F, Godsland IF, Ghatei M, Proudler AJ, Aldis S, Walton C, Bloom S, Stevenson JC: Hyperleptinemia as a component of a metabolic syndrome of cardiovascular risk. Arterioscler Thromb Vasc Biol 1998, 18:928-933.

23. Maslowska M, Vu H, Phelis S, Sniderman AD, Rhode BM, Blank D, Cianflone K: Plasma acylation stimulating protein, adipsin and lipids in non-obese and obese populations. Eur J Clin Invest 1999, 29:679-686.

24. Meijssen S, van Dijk H, Verseyden C, Erkelens DW, Cabezas MC: Delayed and exaggerated postprandial complement component 3 response in familial combined hyperlipidemia. Arterioscler Thromb Vasc Biol 2002, 22:811-816.

25. Murray I, Sniderman AD, Cianflone K: Mice lacking acylation stimulating protein (ASP) have delayed postprandial triglyceride clearance. J Lipid Res 1999, 40:1671-1676.

\section{doi:10.1186/1758-5996-6-132}

Cite this article as: Lopes et al:: Influence of acute hyperlipidemia to adipocyte-derived hormones in lean normotensive and subjects with metabolic syndrome. Diabetology \& Metabolic Syndrome 2014 6:132. 